\title{
Electroacupuncture ameliorates motor dysfunction via inhibiting p66Shc-mediated oxidative stress and endoplasmic reticulum stress in rats with spinal cord injury
}

\section{Fusheng Zhao}

Department of physiology, School of Basic Medical Science, Mudanjiang Medical University, Tongxiang Road 3, Mudanjiang 157011, Heilongjiang, China

\section{Geng Wu}

Department of Diagnostics, School of Clinical Medicine, Mudanjiang Medical University, Tongxiang Road 3, Mudanjiang 157011, Heilongjiang, China

\section{Yang Wu}

Department of Ultrasound, Mudanjiang Medical University Affiliated Hongqi Hospital, Tongxiang Road 3, Mudanjiang 157011, Heilongjiang, China

\section{Yunlong Bai}

School of Basic Medical Science, Jiamusi University, Xuefu Street 258, Jiamusi 154000, Heilongjiang, China

\section{Yongjia Qiu}

Department of physiology, School of Basic Medical Science, Mudanjiang Medical University, Tongxiang Road 3, Mudanjiang 157011, Heilongjiang, China

\section{Wen Lei}

Department of physiology, School of Basic Medical Science, Mudanjiang Medical University, Tongxiang Road 3, Mudanjiang 157011, Heilongjiang, China

Yanzhong Guan ( $\square$ yanzhongguanmdj@sina.com )

Mudanjiang Medical University

\section{Research}

Keywords: Spinal cord injury, Electroacupuncture, Oxidative stress, P66Shc, Endoplasmic reticulum stress

Posted Date: May 13th, 2020

DOI: https://doi.org/10.21203/rs.3.rs-26798/v1

License: (9) This work is licensed under a Creative Commons Attribution 4.0 International License.

Read Full License 
Page 2/24 


\section{Abstract}

Background: Spinal cord injury (SCl) is a severe neurological disorder for which there is currently no effective treatment. Electroacupuncture (EA) is a means of combining traditional acupuncture with modern electrotherapy, which has been widely used and verified to have neuroprotective effects. The aim of this study was to evaluate the effects of EA treatment on the repair of SCl and to investigate the possible mechanisms.

Methods: Rats were randomly divided into sham, sham+EA, SCl and SCl+EA four groups after SCl model was established. Rat motor function was assessed by the Basso, Beattie and Bresnahan locomotor rating scale, inclined plane test and footprint analysis. Histological alterations were examined with hematoxylineosin and Nissl staining. Oxidative stress was evaluated by measuring reactive oxygen species (ROS), glutathione (GSH), total antioxidant capacity (T-AOC), 3-nitrotyrosine (3-NT), as well as 4-hydroxynonenal (4-HNE) levels. The expression of p66Shc and endoplasmic reticulum stress (ERS) were detected to explore the involved mechanisms.

Results: EA treatment significantly improved motor functional recovery, reduced spinal cord lesion cavity and neuronal chromatolysis after SCl. Meanwhile, EA treatment alleviated oxidative stress, as indicated by suppression of ROS production, increase in GSH and T-AOC levels and reduction of 3-NT and 4-HNE expression. Further, EA stimulation markedly eliminated the aberrant increase of p66Shc due to $\mathrm{SCl}$ in rats. More notably, EA treatment also attenuated ERS via down-regulation of glucose-regulated protein 78 , activating transcription factor 4, C/EBP homologous protein, $\mathrm{X}$-box binding protein 1 and activating transcription factor 6 expression in rat spinal cord tissues after SCl.

Conclusions: These findings suggest that $\mathrm{EA}$ is a potential strategy for treatment of $\mathrm{SCl}$, and the mechanism might be, at least in part, associated with mitigation of p66Shc-mediated oxidative stress and ERS in rats.

\section{Background}

Spinal cord injury (SCl) is a consequence of acute traumatic insults of the neural components in spinal canal, which causes degeneration of axons, and induces necrosis or apoptosis of neurons as well as glial cells, and in turn leads to motor, sensory and autonomic dysfunctions in patients with SCI [1]. Although multiple therapeutic strategies have been developed to treat SCl, the short-and long-term effects of the treatments are not ideal [2]. It is well established that the pathophysiology of acute SCl involves two stages: the primary injury that is the initial mechanical damage; and the subsequent secondary injury that results from a progressive local cascade of tissue destruction, including ischemia, edema, calcium overload, inflammation, oxidative stress, as well as neuronal and glial apoptosis, which spread beyond the initial mechanical injury site and lead to irreversible paraplegia and quadriplegia [3].

Lines of evidence indicates that among many pathogenic factors oxidative stress may play a pivotal role in the pathophysiology of SCI [1]. Oxidative stress refers to an imbalance of oxidant/antioxidant systems 
with a progressive accumulation of reactive oxygen species (ROS) and the deficient antioxidants within cells [4]. Under physiological concentrations, ROS are essential for life, as they participate in bactericidal activity of phagocytes, and involve in signal transduction, cell growth and immune reactions.

Nevertheless, excessive ROS generation may damage the cellular components including proteins, lipids, carbohydrates as well as nucleic acids, and consequently result in a wide variety of diseases such as SCI [3]. Normally, intracellular ROS can be elevated though three mechanisms: reducing ROS scavenging, increasing membrane oxidase activity, and by mitochondrial respiratory chain leakage [5]. Accumulation of evidence suggests that p66Shc is involved in the three processes [6]. P66Shc is encoded by the ShcA gene locus that is expressed as three isoforms of about 46, 52 and $66 \mathrm{kDa}$ in mammals [7]. Studies have shown that p66Shc plays a crucial role in regulating signal transduction for cellular oxidative stress, and is one key protein in body for mitochondrial oxidative metabolism and cellular oxidative injury $[4,8]$. A previous study revealed that p66Shc-deficient in mice was resistant to apoptosis under oxidative stress, and extended mice life span [9]. By contrast, several surveys have manifested that higher level of p66Shc in vascular endothelial cells and peripheral blood monocytes is the critical factor inducing oxidative stress in diabetic mice model and diabetic patients [10-12].There is evidence that p66Shc-mediated oxidative stress involves in the activation of the endoplasmic reticulum stress (ERS) response, which leads to cellular malfunction and even cell death [13].

Endoplasmic reticulum (ER), a complicated cytoplasm organelle, is the site for protein synthesis and calcium homeostasis. A growing body evidence indicates that oxidative stress may impair ER function and promote ERS, which results in various diseases, such as diabetes, traumatic injury and neurodegeneration [14-16]. Upon ERS, accumulation of misfolded or unfolded proteins induce activation of three specific stress sensors: protein kinase RNA-like ER kinase (PERK), inositol-requiring enzyme 1 (IRE1) and activating transcription factor 6 (ATF6) [17]. IRE1 and ATF6 are mainly mediated by ER molecular chaperones, such as glucose-regulated protein 78 (GRP78), thereby promoting the capacity of protein folding. The activation of PERK facilitates the eukaryotic translation initiation factor 2 subunit a (elF2a) phosphorylation and enables ER to recover from the stressed condition [18]. Moreover, prolonged and uncontrolled ERS also activates C/EBP homologous protein (CHOP), a downstream regulating protein of the PERK pathway, resulting in cell death and apoptosis [19].

As one of the traditional oriental medicines, acupuncture has been used as a therapeutic technique for thousands of years to treat various types of diseases [20]. Electroacupuncture (EA) is a type of traditional acupuncture modalities in which an electrical current is applied to acupuncture needles while they have been inserted into specific points in the body called acupoints [21]. Clinically, EA has been rendered as a valid treatment therapy for many neurological disorders, such as anxiety disorders and traumatic brain injury $[22,23]$. In animal experiments, repeated EA stimulations have been shown to ameliorate cognitive impairment in app/ps1 transgenic mice, and to relief pain in a rat model of postoperative ileus [24, 25]. In addition, EA treatment has been reported to attenuate blood-brain barrier disruption via regulation of oxidative stress in rats after ischemic stroke [26]. While reviewing the literature reports, we discovered that Dazhui (GV14) and Mingmen (GV4) were the acupoints that frequently used for treatment neurological disorders in clinical or animal models $[27,28]$. The GV14 is the confluence of Governor Vessel and the 
hand and foot three Yang meridians. Acupuncture stimulation of GV14 is believed to activate channel-Qi and involved in the improvement of headache, dizziness, insomnia, forgetfulness, cerebral thrombosis as well as cerebral infarction [28-30]. The GV4 is recognized the root of Yuan-Qi and the gateway of human body that implicates in adjustment of collaterals and strengthens the spine and kidneys functions [31, 32].

In view of the above considerations, the present study aimed to investigate whether EA treatment could ameliorate $\mathrm{SCl}$ and, if so, whether the protective effects were associated with alleviation of p66Shcraleated oxidative stress and ERS in rats.

\section{Materials And Methods}

\section{Animals and experimental groups}

Sprague-Dawley rats (4-6 weeks old, 48 males and 48 females) were obtained from the Experimental Animal Centre of Mudanjiang Medical University of China. The rats were maintained under specificpathogen-free conditions at $22 \pm 2{ }^{\circ} \mathrm{C}$ and $45 \pm 5 \%$ humidity with ad libitum access to food and water. After 7 days acclimation, rats were randomly assigned into 4 groups (24 rats per group): (1) sham group, rats only received a laminectomy; (2) sham + EA group, rats subjected to a laminectomy and treated with EA at GV14 and GV4 acupoints; (3) SCl group, rats underwent spinal cord hemisection injury at the T9 segment; (4) SCI + EA group, rats received EA treatment at GV14 and GV4 acupoints following spinal cord hemisection injury. All animal experimental procedures were carried out in strict accordance with the recommendation in the National Institute of Health Guide for the Care and Use of Laboratory Animals (NIH publications No.8023) revised 1978, and approved by the Animal Care and Use Committee of Mudanjiang Medical University. All efforts were made to minimize the number of animals used and their suffering.

\section{Spinal cord hemisection injury}

The spinal cord hemisection injury was produced using the protocol described previously [33]. Briefly, rats were anesthetized using $2 \%$ pentobarbital sodium ( $40 \mathrm{mg} \mathrm{kg}^{-1}$; Solarbio Science \& Technology, Beijing, China) by intraperitoneal injections, and an incision was made at the dorsal midline, followed by cutting the cutaneous tissue and separating the muscle. Then, the laminectomy was performed at the T8 vertebral level. After the dura had been exposed, a hemisection was conducted using an iridectomy scissors at T9 spinal segment and with no tissue removed. The tail was spastically swung and the posterior limb twitching rigidity confirming that the hemisection of the spinal cord was completed. Rats in the sham and sham + EA groups were subjected to a laminectomy without spinal cord hemisection injury. After adequate hemostasis with absorbable gelatin sponge, the musculature, fascia and skin were sutured in layers. During the procedures, rat temperature was recorded and maintained at $37^{\circ} \mathrm{C}$ using a heating pad. After surgery, rats received an intramuscular injection of penicillin $(100,000 \mathrm{U} / \mathrm{d})$ for 3 days and placed on thick soft bedding in separated cages with sufficient water and food. 


\section{Acupuncture administration}

EA treatment was performed once daily at GV14 and GV4 acupoints. According to the Laboratory Animal Acupuncture Atlas developed by the National Acupuncture Society for Experimental Research, GV14 is located on the posterior midline below the spinous process of the seventh cervical vertebra, and GV4 is located on the posterior midline below the spinous process of the second lumbar vertebra [31, 34]. Rats were placed within a specially designed cloth bag without anesthesia during EA treatment. Rats in the sham and $\mathrm{SCl}$ groups were also immobilized in a manner same as in the EA groups for duration of 30 minutes, to minimize any discrepancies due to stress. Sterilized disposable stainless steel needles ( $0.30 \mathrm{~mm}$ in diameter, $25 \mathrm{~mm}$ in length, Beijing Zhongyan Taihe Medical Instrument Co., Ltd., Beijing, China) were punctured into GV14 (oblique downwardly) and GV4 (oblique upwardly) as deep as $5 \mathrm{~mm}$ for both points. Then, the needles were connected to the output terminals of an EA apparatus (G6805区electric pulse stimulator, Qingdao Xinsheng Co., Ltd., Qingdao, China), and the electric simulation parameters were set at a frequency of $2 \mathrm{~Hz}$ and an intensity of $1 \mathrm{~mA}$. EA stimulation started on the second day post-surgery and lasted for 56 days.

\section{Motor function assessment}

The Basso, Beattie and Bresnahan (BBB) locomotor rating scale [35] was used to evaluate rat hind limb motor function at $0,3,7,14,21,28,35,42,49$ and 56 days after SCl. Briefly, rats were allowed to walk feely in an open field $(80 \times 130 \times 30 \mathrm{~cm})$ with a pasteboard-covered non-slippery floor. In each testing session, rats were observed individually for $5 \mathrm{~min}$ by two observers who were blinded to grouping. The BBB rating scale ranges from 0 to 21 points, in which a score of 0 indicates complete paralysis and a score of 21 implies normal motor function. The scores were recorded for analysis. The inclined plane test was performed as previously described [34]. In brief, rats were placed with their body axis perpendicular to an orientation of the plate covered with a rubber mat containing horizontal ridges spaced $3 \mathrm{~mm}$ apart, and evaluate the maximum vertical axis of the inclined plate. The maximum angle at which a rat maintained its position for $5 \mathrm{sec}$ without falling was recorded for analysis. Footprint analysis was carried out as previously described [35]. In short, the animal's forepaws and hindpaws were brushed with red and blue nontoxic dye, respectively. Then, the rats were required to walk across a paper lined runway ( 3 feet long, 3 inches wide) to obtain an edible treat in a darkened box at the end, and the footprints were scanned for analysis.

\section{Sample collection and processing}

At 56 days after behavioral tests, rats were sacrificed with $2 \%$ pentobarbital sodium ( $40 \mathrm{mg} \mathrm{kg}^{-1}$; i.p.), and approximately a $0.5 \mathrm{~cm}$ segment of spinal cord centered at the injury epicenter was dissected. Then, the spinal cord segments were immediately frozen in liquid nitrogen and stored at $-80^{\circ} \mathrm{C}$ for fluorescent staining, or fixed in $4 \%$ paraformaldehyde for histological examination, or placed in RNA Later for quantitative real-time polymerase chain reaction (qRT-PCR), or frozen in liquid nitrogen for western blot and biochemical analysis.

\section{Histological examination}


After fixed in 4\% paraformaldehyde overnight, the spinal cord segments were processed and embedded in paraffin, followed by preparation of transverse sections with $5 \mu \mathrm{m}$ thick at the injury epicenter. The spinal cord segments were cut at intervals of $50 \mu \mathrm{m}$. For each level, two sections were obtained. One section was stained with hematoxylin-eosin according to standard techniques, and observed under a light microscope (DM2000, Leica Microsystems, Wetzlar, Germany). The cavity sizes of each section were measured manually with Image Pro Plus 6.0 imaging software (Media Cybernetics UK, Marlow, UK) by an observer blind to the groups, as previously described [36].

The other section was incubated in $0.1 \%$ Cresyl violet Nissl staining solution (Sigma-Aldrich, St. Louis, MO, USA) according to the manufacturer's instructions. The sections were examined with a light microscope, and images were captured with an Mshot color video camera (MD50, Beijing, China).

Neuronal chromatolysis, defined as dissolution or loss of Nissl bodies, was evaluated. The chromatolytic and total neurons were counted, and the proportions of chromatolytic neurons versus total neurons were calculated in each group. Integrated optical density (OD) of Nissl bodies in each section was analyzed as previous study described [37].

\section{Intracellular ROS staining}

Intracellular ROS was measured by using a fluorescent dye dihydroethidium (DHE) commercial kit (keyGEN BioTECH, Nanjing, China) in accordance with the manufacturer's instruction [38]. Briefly, the DHE $(5 \mathrm{mg}$ ) were dissolved in $3.17 \mathrm{~mL}$ dimethylsulfoxide to make a $5 \mathrm{mM}$ DHE reagent stock solution. Then the DHE reagent stock solution was diluted in PBS to make a $50 \mu \mathrm{M}$ DHE reagent working solution. The frozen spinal cord segments were transversely sectioned at $14 \mu \mathrm{m}$, and incubated with DHE reagent working solution for $30 \mathrm{~min}$ at $37^{\circ} \mathrm{C}$ in a humidified chamber protected from light, and then fluorescent images were captured using a fluorescence microscope (Olympus BX51TR, Olympus Corp., Tokyo, Japan). All imaging parameters were kept constant during imaging. Fluorescent intensity was quantified with Image Pro-Plus 6.0 software as previous study described [39].

\section{Glutathione and total antioxidant capacity assay}

The contents of reduced glutathione (GSH) in spinal cord homogenates were measured using a GSH assay kit (Nanjing Jiancheng Bioengineering Institute, Nanjing, China) following the manufacturer's instructions. Briefly, spinal cord segment was homogenized independently in ice cold phosphate buffer and centrifuged at $3500 \mathrm{rpm}$ for $10 \mathrm{~min}$ at $4{ }^{\circ} \mathrm{C}$, the supernatants were collected and incubated with a reaction mixture for $10 \mathrm{~min}$ at $37^{\circ} \mathrm{C}$, and then the GSH contents were assayed by colorimetric analysis. Samples were normalized for differences in the amount of protein as determined by a BCA Protein Assay Kit (Beyotime, Nanjing, China).

Total antioxidant capacity (T-AOC) was measured with a T-AOC assay kit (Nanjing Jiancheng Bioengineering Institute, Nanjing, China) according to the manufacturer's instructions. In brief, the spinal cord homogenates were fully blend and kept still for $10 \mathrm{~min}$, and then the supernatants were collected and measured spectrophotometrically at $520 \mathrm{~nm}$. The protein concentration for each sample was determined by a BCA Protein Assay Kit. 


\section{Enzyme-linked immunosorbent assay}

The levels of 8-hydroxy-2'-deoxyguanosine (8-OHdG) in spinal cord tissues were assessed using a commercial ELISA kit (Wuhan USCN Business Co., Ltd, Wuhan, China) according to the manufacturer's instructions. Briefly, the spinal cord segment was homogenized in RIPA buffer (Beyotime, Nanjing, China), centrifuged at 12,000 rpm for $10 \mathrm{~min}$, and the protein concentrations were measured by BCA Protein Assay Kit. Subsequently, the supernatants were added to 96 -well ELISA plates and incubated at $37^{\circ} \mathrm{C}$ for $30 \mathrm{~min}$. The conjugate reagent was added to the plates. After rinsing, the plates were incubated with color agent at $37^{\circ} \mathrm{C}$ for $30 \mathrm{~min}$, and then the reaction was terminated by stop buffer. The OD was measured with a microplate reader at $450 \mathrm{~nm}$. The levels of 8-OHdG in the samples were calculated based on a standard curve, and the results were reported as $\mathrm{pg} / \mathrm{mg}$ protein.

\section{Quantitative real-time polymerase chain reaction analysis}

The qRT-PCR analysis was conducted following the method described in previous work [39]. Briefly, total RNA was isolated from one half of the spinal cord segment (the spinal cord segment was bisected longitudinally along the middle line) by using TRIzolß reagent (Invitrogen, Thermo Fisher Scientific, Inc., CA, USA), and the RNA concentration and purity were quantified by a spectrophotometer (NanoDrop 2000, Thermo Fisher Scientific, Waltham, MA, USA). The RNA was reverse transcribed into cDNA using Thermo Scientific Revert Aid First Strand cDNA kit (Thermo Scientific, Waltham, MA, USA) according to the manufacturer's instructions. The qRT-PCR was performed using SsoFast EvaGreen ${ }^{\circledR}$ Supermix (Bio-Rad Laboratories, Hercules, CA, USA) with a PCR Detection System (Applied Biosystems, ABI Prism 7300, USA). The final volume of the PCR reaction mixture was $20 \mu \mathrm{L}$, which consisted of $10 \mu \mathrm{L} 2 \times$ SYBR supermix, $0.5 \mu \mathrm{L} 10 \mu \mathrm{M}$ forward primer and reverse primer, $1 \mu \mathrm{L}$ cDNA and $8 \mu \mathrm{L}$ Rnase/DNase-free water. The qRT-PCR was conducted as follows: $95^{\circ} \mathrm{C}$ for $30 \mathrm{sec}$, followed by 40 cycles of $95^{\circ} \mathrm{C}$ for $5 \mathrm{sec}$ and $60^{\circ} \mathrm{C}$ for $5 \mathrm{sec}$. The quantitation values of target genes were analyzed by using the relative quantification method. Briefly, a comparative quantification cycle (Cq) was used to determine gene expression level relative to the endogenous $\beta$-actin control gene. Amplification specificity was checked using melting curves. The relative change in gene expression level was calculated using the $2^{-\triangle \Delta C q}$ values of the different groups. The primers for $p 66 S h c$ as follows: forward, 5'-GCCGAGTATGTCGCCTATGT-3'; reverse, 5'-GGGTGGGTTCCTGAGGTATT-3'; Primer pairs for $\beta$-actin were as follows: forward, 5 'GGTGAAGGTCGGAGTCAACG-3'; reverse, 5'-CCAGTAGGTACTGTTGAAAC-3'.

\section{Western blot analysis}

The western blot analysis was performed as previous study described [39]. Briefly, the remaining half of spinal cord segment was homogenized in RIPA buffer with 1\% phenylmethylsulfonyl fluoride (Beyotime, Nanjing, China) and protease inhibitor (Sigma Aldrich, St Louis, MO, USA) according to the manufacturer's instructions. Samples were centrifuged at 12,000 rpm for $10 \mathrm{~min}$, and then the protein concentrations were determined by BCA Protein Assay Kit. For electrophoresis, protein samples $(30 \mu \mathrm{g})$ were dissolved in the loading buffer and boiled for $5 \mathrm{~min}$. Samples were then resolved on 12\% SDS-PAGE and transferred to PVDF membranes (Millipore, Billerica, MA, USA). The membranes were blocked with $5 \%$ skim milk in 
TBST (10 mmol/L Tris, $150 \mathrm{mmol} / \mathrm{L} \mathrm{NaCl}$ and $0.1 \%$ Tween 20, pH7.5) for $2 \mathrm{~h}$ at $37^{\circ} \mathrm{C}$. Subsequently, the membranes were incubated with primary antibodies at $4{ }^{\circ} \mathrm{C}$ overnight. The primary antibodies were as follows: 3-nitrotyrosine (3-NT, 1:1000), 4-hydroxynonenal (4-HNE, 1:1000) and ATF6 (1:1000) (Abcam, Cambridge, MA, USA); p66Shc (1:1500), GRP78 (1:3000), CHOP (1:1000), X-box binding protein 1 (XBP1, 1:800), ATF4 (1:1000) and $\beta$-actin (1:5000) (Proteintech, Chicago, IL, USA). After three washes in TBST, the membranes were then incubated for $2 \mathrm{~h}$ with HRP-conjugated secondary antibodies (Santa Cruz Biotechnology, Inc., Dallas, TX, USA) at room temperature. Following rinsing with TBST, the target proteins were visualized with chemiluminescence luminol reagents (Millipore, Billerica, MA, USA). Blots were imaged by Molecular Image ${ }^{\circledR}$ ChemiDocTM XRS ${ }^{+}$with Image Lab ${ }^{\text {TM }}$ Software (Bio-Rad, Hercules, CA, USA). The $\beta$-actin served as an internal control. The gray values of bands were analyzed by Image J 1.50 b Gel Analyzer (National Institutes of Health, Washington, DC, USA). The gray values of target proteins were divided by that of internal control to correct the error, which resulted due to the relative content of the target protein in the sample.

\section{Statistical analysis}

All data were presented as mean \pm SEM. A multifactorial analysis of variance (ANOVA) for repeated measurement was employed for analyzing BBB scores and maximum angles of the inclined plane test. One-way ANOVA was employed for analyzing other data followed by LSD (equal variances assumed) or Dunnett's T3 (equal variances not assumed) post hoc test with SPSS 17.0 software package. Statistical significance was set at $P<0.05$.

\section{Results}

\section{EA treatment improves motor functional recovery after SCI in rats}

To confirm whether EA treatment could improve rat locomotion recovery after SCl, the BBB locomotion scale, inclined plane test and footprint recording were performed. As shown in Fig. 1a, the BBB scores of rats in the $\mathrm{SCl}$ group were significantly lower than their counterparts in the sham group at different time points after $\mathrm{SCl}(P<0.05)$. Within 7-21d following EA stimulation, the BBB scores trended to increase in the $\mathrm{SCl}+\mathrm{EA}$ group, however, the difference was not statistically significant compared with the $\mathrm{SCl}$ group $(P>0.05)$. Twenty-eight days after EA stimulation, the BBB scores in the $\mathrm{SCl}+\mathrm{EA}$ group were higher than those in the SCl group $(P<0.05)$, even though it was not restored to the level of the sham group $(P<0.05)$. Additionally, there was no significant difference between the sham and sham + EA groups in terms of BBB scores at different time points $(P>0.05)$. Consistent with the results of BBB assay, the maximum angles of inclined plane test were markedly decreased in rats of the $\mathrm{SCl}$ group compared with those in the sham group at different time points, whereas the decrease was diminished after 28 days of EA stimulation in the $\mathrm{SCl}+\mathrm{EA}$ group $(P<0.05)$, even though it was not restored to the level of the sham group $(P<0.05)$. In addition, the maximum angles were scarcely significantly different between the sham and sham $+E A$ groups $(P>0.05)$ (Fig. 1b). As illustrated in Fig. 1c, footprint analyses revealed that rats from the $\mathrm{SCl}+\mathrm{EA}$ 
group displayed fairly consistent hind limb coordination and very little toe dragging following EA stimulation. By contrast, rat footprints obtained from the $\mathrm{SCl}$ group showed inconsistent coordination and extensive drags as indicated by ink streaks. These results suggested that EA treatment could improve motor functional recovery after $\mathrm{SCl}$ in rats.

\section{EA treatment ameliorates histological alterations after $\mathrm{SCl}$ in rats}

To test whether EA treatment could attenuate spinal cord damage, $\mathrm{HE}$ and Nissl staining was carried out at 56 days after $\mathrm{SCl}$. As shown in Fig. 2a and e, the spinal cord tissues of rats in the sham group were characterized by a clear boundary between the gray and white matter, and the neurons were evenly distributed with normal morphology; the white matter fibers were evenly distributed with neat arrangement. Tissue structures of spinal cords of rats in the sham + EA group (Fig. $2 b$ and $f$ ) were similar to the structures showed in the sham group. In the SCl group (Fig. $2 \mathrm{c}$ and g), the boundaries between gray and white matters became obscured, the neurons were swollen, and the cystic cavities were evident in the injury epicenter. Moreover, a portion of neurons were found with condensed nuclei and vacuolar degeneration. The spinal histological structures in the $\mathrm{SCl}+\mathrm{EA}$ group (Fig. $2 \mathrm{~d}$ and $\mathrm{h}$ ) were relatively complete, and only a few cavities were observed in the gray and white matter. Meanwhile, the neurons were evenly distributed in the gray matter and nerve fibers were regularly arranged in white matter. Quantitative analysis revealed that the cavity area in the $\mathrm{SCl}+\mathrm{EA}$ group was significantly reduced relative to the $\mathrm{SCl}$ group $(P<0.05)$, even though it was not restored to the level of the sham group $(P<0.05)$. ) (Fig. 2q).

Nissl staining showed that neurons in the sham (Fig. 2i and m) and sham + EA (Fig. 2j and n) groups were integrative and the cytoplasm was deeply stained with granular-like morphology, and only a few chromatolytic neurons were observed. In the SCl group (Fig. 2k and o), by contrast, a large number of neurons were dimly stained with chromatolytic appearance. Following EA stimulation, the loss of Nissl bodies was effectively improved in the SCl+EA group (Fig. 2 l and p). As shown in Fig. 2r, the proportion of chromatolytic neurons in the $\mathrm{SCl}$ group was significantly higher than that in the sham group $(P<0.05)$. However, the proportion was reduced in the $\mathrm{SCl}+\mathrm{EA}$ group compared with that in the $\mathrm{SCl}$ group $(P<0.05)$, even though it was not restored to the level of the sham group $(P<0.05)$. In addition, the proportions were scarcely significantly different between the sham and sham + EA groups $(P>0.05)$. In order to evaluate neuronal Nissl body density, we also analyzed the OD values of Nissl staining (Fig. 2s). Compare with the sham group, the OD value was significantly decreased in the SCI group $(P<0.05)$. With EA treatment, the $\mathrm{OD}$ value of the $\mathrm{SCl}+\mathrm{EA}$ group were increased compared with that in the $\mathrm{SCl}$ group $(P<0.05)$, even though it was not restored to the level of the sham group $(P<0.05)$, and there was no significant difference in OD values between the sham and sham + EA groups $(P>0.05)$. These results indicated that EA treatment could ameliorate histological alterations due to $\mathrm{SCl}$ in rats.

\section{EA treatment alleviates oxidative stress after $\mathrm{SCl}$ in rats}


To determine the effect of EA treatment on oxidative stress induced by SCl, the ROS in spinal tissues was initially detected with ROS Fluorescent Probe-DHE (Fig. 3a). As shown in Fig. 3b, the mean fluorescence intensity of DHE in the $\mathrm{SCl}$ group was significantly increased compared with that in the sham group $(P<$ 0.05), whereas the increased fluorescence intensity due to $\mathrm{SCl}$ was reduced with $\mathrm{EA}$ stimulation in the $\mathrm{SCl}$ + EA group $(P<0.05)$, and there was no significant difference in levels of fluorescence intensity between the sham and sham + EA groups $(P>0.05)$. To elucidate whether EA stimulation contributed to change of antioxidant capacity, we subsequently measured the levels of GSH and T-AOC in spinal cord tissues. As illustrated in Fig. 3c, the content of $\mathrm{GSH}$ was strikingly reduced in the $\mathrm{SCl}$ group compared with that in the sham group $(P<0.05)$. In contrast to the $\mathrm{SCl}$ group, however, the decrease in $\mathrm{GSH}$ was reversed following EA stimulation in the $\mathrm{SCl}+\mathrm{EA}$ group $(P<0.05)$, and there was no significant difference in $\mathrm{GSH}$ content between the sham and sham + EA groups $(P>0.05)$. As displayed in Fig. 3d, the level of T-AOC in the SCI group was remarkably lower than that in the sham group $(P<0.05)$. With EA treatment, the T-AOC content in the $\mathrm{SCl}+\mathrm{EA}$ group was elevated compared to the $\mathrm{SCl}$ group $(P<0.05)$. No significant difference was found in T-AOC between the sham and sham + EA groups $(P>0.05)$.

To further confirm the effect of EA treatment on oxidative stress after SCl, the levels of 8-OHdG, 3-NT and 4-HNE in rat spinal tissues were evaluated. As shown in Fig. 3e, the mean level of 8-OHdG was significantly increased in spinal tissues of the rats subjected to $\mathrm{SCl}$ as compared with those in the sham group $(P<0.05)$. Following EA stimulation, the level of 8-OHdG was diminished in the $\mathrm{SCl}+\mathrm{EA}$ group compare with that in the $\mathrm{SCl}$ group $(P<0.05)$, and no significant difference was found in 8-OHdG levels between the sham and sham + EA groups $(P>0.05)$. Furthermore, the results showed that the level of $3-$

NT in the $\mathrm{SCl}$ group was significantly enhanced compared with that in the sham group $(P<0.05)$, whereas the increase was lowered following EA stimulation in the SCI + EA group $(P<0.05)$, and the level of 3-NT did not differ significantly between the sham and sham + EA groups $(P>0.05)$ (Fig. 3f). Likewise, the level of 4-HNE was also elevated in the $\mathrm{SCl}$ group compared with that in the sham group $(P<0.05)$. However, the increase was reversed with EA stimulation in the $\mathrm{SCl}+\mathrm{EA}$ group $(P<0.05)$, and no significant difference was observed in 4-HNE expression between the sham and sham + EA groups $(P>0.05)$ (Fig. 3g). Taken together, these results indicated that EA treatment could alleviate oxidative stress induced by $\mathrm{SCl}$ in rats.

\section{EA treatment down-regulates p66Shc expression after SCI in rats}

To investigate whether the anti-oxidative effect of EA treatment was correlated with p66Shc, a key regulator of cellular oxidative stress, we further analyzed p66Shc expression in rat spinal cord tissues. As showed in Fig. 4a and b, the mRNA and protein expression levels of p66Shc in the $\mathrm{SCl}$ group were notably increased when compared with those in the sham group $(P<0.05)$. Following EA stimulation, however, the mRNA and protein expressions of p66Shc were significantly decreased in the $\mathrm{SCl}+\mathrm{EA}$ group compared with those in the $\mathrm{SCl}$ group $(P<0.05)$. In addition, there was no significant difference between the sham and sham + EA groups in terms of p66Shc expression $(P>0.05)$. These results suggested that EA treatment could down-regulate p66Shc expression after SCl in rats. 


\section{EA treatment suppresses ER stress after $\mathrm{SCl}$ in rats}

To further explore the potential mechanism of EA treatment against SCI, ER stress was evaluated. As shown in Fig. 5a-e, the protein expression levels of GRP78, ATF4, CHOP as well as XBP1 and ATF6 were significantly increased in rat spinal cord tissues after $\mathrm{SCl}$ as compared with those in the sham group ( $P<$ 0.05). After EA stimulation, the levels of these ER stress-related proteins in the $\mathrm{SCl}+\mathrm{EA}$ group were decreased compared with those in the $\mathrm{SCl}$ group $(P<0.05)$. In addition, there was no significant difference in terms of GRP78, ATF4, CHOP, XBP1 and ATF6 expressions between the sham and sham + EA groups $(P>0.05)$. These results indicated that EA treatment could attenuate ER stress after $\mathrm{SCl}$ in rats.

\section{Discussion}

In this study, we provided evidence that EA stimulation on GV14 and GV4 acupoints could promote motor functional recovery, reduce spinal cord lesion cavity and attenuate neuronal chromatolysis after SCl. Furthermore, EA stimulation could alleviate oxidative stress and eliminated the aberrant increase of p66shc due to $\mathrm{SCl}$ in rats. Notably, EA stimulation was also able to attenuate ER stress, as indicated by down-regulating GRP78, ATF4, CHOP, XBP1 and ATF6 expression after SCl. Thus, our findings demonstrated that EA treatment could ameliorate motor dysfunction after $\mathrm{SCl}$, which might be partly associated with inhibiting p66Shc-mediated oxidative stress and ER stress in rats.

Acupuncture, as one part of the Traditional Chinese Medicine, has been widely practiced in both Eastern Asia and Western countries for treatment of a variety of diseases, such as depression and traumatic brain injury $[22,40]$. Studies in animal models demonstrate that EA stimulation exerts neuroprotective effects by inhibiting cell apoptosis and improving synaptic plasticity in cerebral ischemia-reperfusion injury [41, 42]. Consistent with these studies, we discovered in the present study that EA stimulation could improve rat motor function recovery after $\mathrm{SCl}$. Moreover, we found that EA treatment was also able to attenuate spinal cord histological damage after $\mathrm{SCl}$. These findings raise questions regarding the mechanisms underlying the effect of EA treatment on the repair of SCl.

Evidence from multiple sources revealed that oxidative stress acted as a predominant pathogenic factor that induced the pathological changes after SCl. A previous study indicated that EA stimulation could attenuate oxidative stress and cell apoptosis by decreasing the production of hydrogen peroxide and malonaldehyde in mice with Parkinson's disease [43]. Similarly, the therapeutic effect of EA stimulation was also confirmed in a rat model of Alzheimer's disease induced by A $1-42$ [44]. In the present study, we discovered that EA treatment effectively suppressed ROS production due to $\mathrm{SCl}$ in rats. Several lines of evidence suggests that excessive ROS may directly interact with cellular biomolecules, such as lipids, proteins and nucleic acids, and cause damage to cellular constituents [45]. Within cells, there exists an antioxidant system capable of scavenging ROS, which comprises enzymatic antioxidants including catalase, superoxide dismutase (SOD), guaiacol peroxidase (GPX), as well as non-enzymatic scavengers such as GSH, vitamin $\mathrm{C}$ and vitamin E [46]. Studies have revealed that GSH is a predominant antioxidant that scavenges cellular ROS and other reactive nitrogen species through non-enzymatically reacts with 
radicals in a direct manner or indirectly enzymatic reaction $[47,48]$. Depletion or deficiency of GSH contributes to oxidative tissue damage. A previous study reported that acupuncture at Yanglingquan (GB34), Taichong (LR3), Zusanli (ST36) and Xuehai (SP10) acupoints could enhance GSH content and suppress rotational behavior in 6-hydroxydopamine-lesioned rats [49]. Consist with this findings, our current study discovered that rats subjected to $\mathrm{SCl}$ showed distinct reduce of $\mathrm{GSH}$ in spinal cord tissues. Meanwhile, the T-AOC, a biomarker of total antioxidant status in cells, was also sharply decreased in SCI rats. Interestingly, the decrease in both GSH and T-AOC due to SCI was restored with EA treatment.

To further confirm the anti-oxidative effect of EA treatment, the other indicators of oxidative stress were measured in the current study. The 8-OHdG, a oxidative product form of guanine, is frequently used to quantify the DNA oxidative damage [50]. 4-HNE is a kind of aldehyde produced during lipid peroxidation of unsaturated lipids in cellular membrane. Under condition of oxidative stress, lipid peroxidation alters membrane structure, influencing its fluidity and permeability as well as the activity of membrane-bound proteins, and eventually results in a variety of biological responses, such as cellular dysfunction and apoptosis [51]. 3-NT is formed by oxidation of nitrite, which induces protein denaturation, and involves in diverse pathological changes [52]. In this study, we found that the levels of 8-OHdG, 4-HNE and 3-NT in rat spinal cord tissues were obviously elevated after $\mathrm{SCl}$, however these oxidative damage markers were lowered following EA stimulation. These findings clearly suggested a protective effect of EA stimulation against $\mathrm{SCl}$-induced oxidative damage after $\mathrm{SCl}$.

To explore the possible mechanism of EA against $\mathrm{SCl}$, we detected the expression of p66Shc in rat spinal cord tissues. Accumulating evidence indicates that p66Shc acts as a master regulator of ROS and expresses in many tissues, where it involves in organ dysfunction and multiple diseases [53]. Studies have revealed that mice deletion of p66Shc have a prolonged lifespan and display an increased resistance to oxidative stress [54-57]. In addition, researchers found that p66Shc was able to decrease the expression of antioxidant enzymes such as SOD and GPX by down-regulating forkhead-type transcription factors [53]. Nevertheless, whether EA stimulation can influence p66Shc expression after SCI has never been described. In this study, our data clearly showed that the mRNA and protein levels of p66Shc in rat spinal cord tissues were increased after SCI. Furthermore, we firstly found that the enhanced p66Shc was mitigated when rats received EA treatment. Base on these findings, we speculate that EA stimulation may augment enzymatic and non-enzymatic antioxidants contents and regulate p66Shc expression, and consequently attenuate SCl-induced oxidative stress in rats.

Under many pathological conditions such as hypoxia, environmental toxins, glucose deprivation and ischemia can induce ER stress (ERS), and result in functional alterations and build-up of unfolded proteins in ER [58]. Consequently, the unfolded protein response (UPR) is triggered. There is evidence that the UPR is orchestrated by PERK, IRE1 and ATF6 sensors, and is mediated by GRP78, also known as molecular chaperones. Under ERS conditions, misfolded proteins in ER lumen will induce release of GRP78 from the three sensors that subsequently activate downstream transcription factors such as XBP1, ATF4 and CHOP [58]. In accordance with the opinion, we found in this study that the levels of ERS associated proteins including GRP78, ATF4, CHOP, XBP1 and ATF6 were sharply increased in rats with 
$\mathrm{SCl}$, however, the increase trend was reversed following EA stimulation, which suggested that EA was a potential therapy to alleviate ERS after SCl.

\section{Conclusions}

In summary, the present study demonstrates that EA can ameliorate motor dysfunction caused by $\mathrm{SCl}$, which possibly via inhibiting p66Shc-mediated oxidative stress and ER stress in rats. Although the molecular mechanism remains incompletely clear, our findings suggest that EA may serve as a potential therapeutic approach for the treatment of $\mathrm{SCl}$.

\section{Abbreviations}

SCI: Spinal cord injury; EA: Electroacupuncture; ROS: Reactive oxygen species; GSH: Glutathione; T-AOC: Total antioxidant capacity; 3-NT: 3-nitrotyrosine; 4-HNE: 4-hydroxynonenal; ER: Endoplasmic reticulum; ERS: endoplasmic reticulum stress; UPR: unfolded protein response; PERK: Protein kinase RNA-like ER kinase; IRE1: Inositol-requiring enzyme 1; ATF6: Activating transcription factor 6; GRP78: Glucoseregulated protein 78; elF2a: eukaryotic translation initiation factor 2 subunit a; CHOP: C/EBP homologous protein; XBP1: X-box binding protein 1; BBB: Basso, Beattie, and Bresnahan; OD: Optical density; 8-OHdG: 8-hydroxy-2'-deoxyguanosine.

\section{Declarations}

\section{Ethics approval and consent to participate}

All animal experimental procedures were carried out in strict accordance with the recommendation in the National Institute of Health Guide for the Care and Use of Laboratory Animals (NIH publications No.8023) revised 1978, and approved by the Animal Care and Use Committee of Mudanjiang Medical University.

\section{Consent for publication}

Not applicable.

\section{Availability of data and materials}

Not applicable.

\section{Competing interests}

The authors declare that they have no competing interests.

\section{Funding}

This work was supported by the Traditional Chinese Medicine Research Project of Heilongjiang Province (No.ZHY18-167), the Research Fund of Heilongjiang Provincial Health Committee (No.2019-383) and the 
National Natural Science Foundation of China (No.81871041 \& No.81371463 to YZG).

\section{Authors' contributions}

ZFS, WG, WY, BYL, QYJ and LW carried out all the animal experiments. ZFS and WG participated in the design of the experimental methods. WY and BYL participated in the analysis of the data. ZFS and GYZ designed the study and drafted the manuscript. GYZ designed the study and revised the manuscript. All authors read and approved the final manuscript.

\section{Acknowledgements}

The authors are grateful to Sulian Li, Chunlei Zhang and Xuekui Shi for their technical support and helpful discussion.

\section{Author details}

${ }^{1}$ Department of physiology, School of Basic Medical Science, Mudanjiang Medical University, Tongxiang Road 3, Mudanjiang 157011, Heilongjiang, China.

${ }^{2}$ Department of Diagnostics, School of Clinical Medicine, Mudanjiang Medical University, Tongxiang Road 3, Mudanjiang 157011, Heilongjiang, China.

${ }^{3}$ Department of Ultrasound, Mudanjiang Medical University Affiliated Hongqi Hospital, Tongxiang Road 3, Mudanjiang 157011, Heilongjiang, China.

${ }^{4}$ School of Basic Medical Science, Jiamusi University, Xuefu Street 258, Jiamusi 154000, Heilongjiang, China.

\section{References}

1. Alizadeh A, Dyck SM, Karimi-Abdolrezaee S. Traumatic Spinal Cord Injury: An Overview of Pathophysiology, Models and Acute Injury Mechanisms. Front Neurol. 2019; 10:282.

2. Zadeh-Ardabili PM, Rad SK, Rad SK, Khazaai H, Sanusi J, Zadeh MH. Palm vitamin E reduces locomotor dysfunction and morphological changes induced by spinal cord injury and protects against oxidative damage. Sci Rep. 2017; 7:14365.

3. Fatima G, Sharma VP, Das SK, Mahdi AA. Oxidative stress and antioxidative parameters in patients with spinal cord injury: implications in the pathogenesis of disease. Spinal Cord. 2015; 53:3-6.

4. Xie ZZ, Liu Y, Bian JS. Hydrogen Sulfide and Cellular Redox Homeostasis. Oxid Med Cell Longev. 2016; 2016:6043038.

5. Schieber M, Chandel NS. ROS function in redox signaling and oxidative stress. Curr Biol. 2014; 24:R453-462. 
6. Nemoto S, Finkel T. Redox regulation of forkhead proteins through a p66shc-dependent signaling pathway. Science. 2002; 295:2450-2452.

7. De Marchi E, Baldassari F, Bononi A, Wieckowski MR, Pinton P. Oxidative stress in cardiovascular diseases and obesity: role of p66Shc and protein kinase C. Oxid Med Cell Longev. 2013; 2013:564961.

8. Minami Y, Sonoda N, Hayashida E, Makimura H, Ide M, Ikeda N, et al. p66Shc Signaling Mediates Diabetes-Related Cognitive Decline. Sci Rep. 2018; 8:3213.

9. Ray PD, Huang BW, Tsuji Y. Reactive oxygen species (ROS) homeostasis and redox regulation in cellular signaling. Cell Signal. 2012; 24:981-990.

10. Pagnin E, Fadini G, de Toni R, Tiengo A, Calo L, Avogaro A. Diabetes induces p66shc gene expression in human peripheral blood mononuclear cells: relationship to oxidative stress. J Clin Endocrinol Metab. 2005; 90:1130-1136.

11. Sun L, Xiao L, Nie J, Liu FY, Ling GH, Zhu XJ, et al. p66Shc mediates high-glucose and angiotensin IIinduced oxidative stress renal tubular injury via mitochondrial-dependent apoptotic pathway. Am J Physiol Renal Physiol. 2010; 299:F1014-1025.

12. Zhang M, Lin L, Xu C, Chai D, Peng F, Lin J. VDR Agonist Prevents Diabetic Endothelial Dysfunction through Inhibition of Prolyl Isomerase-1-Mediated Mitochondrial Oxidative Stress and Inflammation. Oxid Med Cell Longev. 2018; 2018:1714896.

13. Cheng YS, Dai DZ, Dai Y. AQP4 KO exacerbating renal dysfunction is mediated by endoplasmic reticulum stress and p66Shc and is attenuated by apocynin and endothelin antagonist CPU0213. Eur J Pharmacol. 2013; 721:249-258.

14. Breton-Romero R, Weisbrod RM, Feng B, Holbrook M, Ko D, Stathos MM, et al. Liraglutide Treatment Reduces Endothelial Endoplasmic Reticulum Stress and Insulin Resistance in Patients With Diabetes Mellitus. J Am Heart Assoc. 2018; 7:e009379.

15. Xiang $\mathrm{C}$, Wang $\mathrm{Y}$, Zhang $\mathrm{H}, \mathrm{Han} \mathrm{F}$. The role of endoplasmic reticulum stress in neurodegenerative disease. Apoptosis. 2017; 22:1-26.

16. Sun D, Chen X, Gu G, Wang J, Zhang J. Potential Roles of Mitochondria-Associated ER Membranes (MAMs) in Traumatic Brain Injury. Cell Mol Neurobiol. 2017; 37:1349-1357.

17. Lin CL, Lee $\mathrm{CH}$, Chen $\mathrm{CM}$, Cheng CW, Chen PN, Ying TH, et al. Protodioscin Induces Apoptosis Through ROS-Mediated Endoplasmic Reticulum Stress via the JNK/p38 Activation Pathways in Human Cervical Cancer Cells. Cell Physiol Biochem. 2018; 46:322-334.

18. Ron D, Walter P. Signal integration in the endoplasmic reticulum unfolded protein response. Nat Rev Mol Cell Biol. 2007; 8:519-529.

19. Huang C, Wang JJ, Ma JH, Jin C, Yu Q, Zhang SX. Activation of the UPR protects against cigarette smoke-induced RPE apoptosis through up-regulation of Nrf2. J Biol Chem. 2015; 290:5367-5380.

20. Zeng XH, Li QQ, Xu Q, Li F, Liu CZ. Acupuncture mechanism and redox equilibrium. Evid Based Complement Alternat Med. 2014;2014:483294. 
21. Jiang SH, Tu WZ, Zou EM, Hu J, Wang S, Li JR, et al. Neuroprotective effects of different modalities of acupuncture on traumatic spinal cord injury in rats. Evid Based Complement Alternat Med. 2014; 2014:431580.

22. Liu J, Xue X, Wu Y, Yang C, Li N, Li H. Efficacy and safety of electro-acupuncture treatment in improving the consciousness of patients with traumatic brain injury: study protocol for a randomized controlled trial. Trials. 2018; 19:296.

23. Amorim D, Amado J, Brito I, Fiuza SM, Amorim N, Costeira C, et al. Acupuncture and electroacupuncture for anxiety disorders: A systematic review of the clinical research. Complement Ther Clin Pract. 2018; 31:31-37.

24. Lin R, Chen J, Li X, Mao J, Wu Y, Zhuo P, et al. Electroacupuncture at the Baihui acupoint alleviates cognitive impairment and exerts neuroprotective effects by modulating the expression and processing of brain-derived neurotrophic factor in APP/PS1 transgenic mice. Mol Med Rep. 2016; 13:1611-1617.

25. Murakami H, Li S, Foreman R, Yin J, Hirai T, Chen JDZ. Ameliorating Effects of Electroacupuncture on Dysmotility, Inflammation, and Pain Mediated via the Autonomic Mechanism in a Rat Model of Postoperative lleus. J Neurogastroenterol Motil. 2019; 25:286-299.

26. Jung YS, Lee SW, Park JH, Seo HB, Choi BT, Shin HK. Electroacupuncture preconditioning reduces ROS generation with NOX4 down-regulation and ameliorates blood-brain barrier disruption after ischemic stroke. J Biomed Sci. 2016; 23:32.

27. Kim YR, Kim HN, Ahn SM, Choi YH, Shin HK, Choi BT. Electroacupuncture promotes post-stroke functional recovery via enhancing endogenous neurogenesis in mouse focal cerebral ischemia. PLoS One. 2014; 9:e90000.

28. Wang X, Shi SH, Yao HJ, Jing QK, Mo YP, Lv W, et al. Electroacupuncture at Dazhui (GV14) and Mingmen (GV4) protects against spinal cord injury: the role of the Wnt/beta-catenin signaling pathway. Neural Regen Res. 2016; 11:2004-2011.

29. Zheng CX, Lu M, Guo YB, Zhang FX, Liu H, Guo F, et al. Electroacupuncture Ameliorates Learning and Memory and Improves Synaptic Plasticity via Activation of the PKA/CREB Signaling Pathway in Cerebral Hypoperfusion. Evid Based Complement Alternat Med. 2016; 2016:7893710.

30. Cheng CY, Lin JG, Tang NY, Kao ST, Hsieh CL. Electroacupuncture-like stimulation at the Baihui (GV20) and Dazhui (GV14) acupoints protects rats against subacute-phase cerebral ischemiareperfusion injuries by reducing S100B-mediated neurotoxicity. PLoS One. 2014; 9:e91426.

31. Geng $X$, Sun T, Li JH, Zhao N, Wang Y, Yu HL. Electroacupuncture in the repair of spinal cord injury: inhibiting the Notch signaling pathway and promoting neural stem cell proliferation. Neural Regen Res. 2015; 10:394-403.

32. Tian WQ, Peng YG, Cui SY, Yao FZ, Li BG. Effects of electroacupuncture of different intensities on energy metabolism of mitochondria of brain cells in rats with cerebral ischemia-reperfusion injury. Chin J Integr Med. 2015; 21:618-623. 
33. Zhang J, Zhao F, Wu G, Li Y, Jin X. Functional and histological improvement of the injured spinal cord following transplantation of Schwann cells transfected with NRG1 gene. Anat Rec (Hoboken). 2010; 293:1933-1946.

34. Mo YP, Yao HJ, Lv W, Song LY, Song HT, Yuan XC, et al. Effects of Electroacupuncture at Governor Vessel Acupoints on Neurotrophin-3 in Rats with Experimental Spinal Cord Injury. Neural Plast. 2016; 2016:2371875.

35. Basso DM, Beattie MS, Bresnahan JC. A sensitive and reliable locomotor rating scale for open field testing in rats. J Neurotrauma. 1995; 12:1-21.

36. Bai L, Mei X, Shen Z, Bi Y, Yuan Y, Guo Z, et al. Netrin-1 Improves Functional Recovery through Autophagy Regulation by Activating the AMPK/mTOR Signaling Pathway in Rats with Spinal Cord Injury. Sci Rep. 2017; 7:42288.

37. Yan X, Lei F, Hu Y, Nie L, Jia Q, Zhou H, et al. Hydrogen sulfide protects neonatal rat medulla oblongata against prenatal cigarette smoke exposure via anti-oxidative and anti-inflammatory effects. Environ Toxicol Pharmacol. 2018; 57:151-158.

38. Liu H, Zhao L, Yue L, Wang B, Li X, Guo H, et al. Pterostilbene Attenuates Early Brain Injury Following Subarachnoid Hemorrhage via Inhibition of the NLRP3 Inflammasome and Nox2-Related Oxidative Stress. Mol Neurobiol. 2017; 54:5928-5940.

39. Zhao F, Lei F, Yan X, Zhang S, Wang W, Zheng Y. Protective Effects of Hydrogen Sulfide Against Cigarette Smoke Exposure-Induced Placental Oxidative Damage by Alleviating Redox Imbalance via Nrf2 Pathway in Rats. Cell Physiol Biochem. 2018; 48:1815-1828.

40. Zeng L, Tao Y, Hou W, Zong L, Yu L. Electro-acupuncture improves psychiatric symptoms, anxiety and depression in methamphetamine addicts during abstinence: A randomized controlled trial. Medicine (Baltimore). 2018; 97:e11905.

41. Lin R, Li X, Liu W, Chen W, Yu K, Zhao C, et al. Electro-acupuncture ameliorates cognitive impairment via improvement of brain-derived neurotropic factor-mediated hippocampal synaptic plasticity in cerebral ischemia-reperfusion injured rats. Exp Ther Med. 2017; 14:2373-2379.

42. Xue X, You Y, Tao J, Ye X, Huang J, Yang S, et al. Electro-acupuncture at points of Zusanli and Quchi exerts anti-apoptotic effect through the modulation of PI3K/Akt signaling pathway. Neurosci Lett. 2014; 558:14-19.

43. Wang $H$, Pan $Y$, Xue B, Wang $X$, Zhao F, Jia J, et al. The antioxidative effect of electro-acupuncture in a mouse model of Parkinson's disease. PLoS One. 2011; 6:e19790.

44. Wu G, Li L, Li HM, Zeng Y, Wu WC. Electroacupuncture ameliorates spatial learning and memory impairment via attenuating NOX2-related oxidative stress in a rat model of Alzheimer's disease induced by Abeta1-42. Cell Mol Biol (Noisy-le-grand). 2017; 63:38-45.

45. Salim S. Oxidative Stress and the Central Nervous System. J Pharmacol Exp Ther. 2017; 360:201205.

46. Chu H, Shi Y, Jiang S, Zhong Q, Zhao Y, Liu Q, et al. Treatment effects of the traditional Chinese medicine Shenks in bleomycin-induced lung fibrosis through regulation of TGF-beta/Smad3 
signaling and oxidative stress. Sci Rep. 2017; 7:2252.

47. Aquilano K, Baldelli S, Ciriolo MR. Glutathione: new roles in redox signaling for an old antioxidant. Front Pharmacol. 2014; 5:196.

48. Xu X, Lv H, Xia Z, Fan R, Zhang C, Wang Y, et al. Rhein exhibits antioxidative effects similar to Rhubarb in a rat model of traumatic brain injury. BMC Complement Altern Med. 2017; 17:140.

49. Yu YP, Ju WP, Li ZG, Wang DZ, Wang YC, Xie AM. Acupuncture inhibits oxidative stress and rotational behavior in 6-hydroxydopamine lesioned rat. Brain Res. 2010; 1336:58-65.

50. Di Minno A, Turnu L, Porro B, Squellerio I, Cavalca V, Tremoli E, et al. 8-Hydroxy-2-Deoxyguanosine Levels and Cardiovascular Disease: A Systematic Review and Meta-Analysis of the Literature. Antioxid Redox Signal. 2016; 24:548-555.

51. Breitzig M, Bhimineni C, Lockey R, Kolliputi N. 4-Hydroxy-2-nonenal: a critical target in oxidative stress? Am J Physiol Cell Physiol. 2016; 311:C537-C543.

52. Ahsan H. 3-Nitrotyrosine: A biomarker of nitrogen free radical species modified proteins in systemic autoimmunogenic conditions. Hum Immunol. 2013; 74:1392-1399.

53. Galimov ER. The Role of p66shc in Oxidative Stress and Apoptosis. Acta Naturae. 2010;2:44-51.

54. Napoli C, Martin-Padura I, de Nigris F, Giorgio M, Mansueto G, Somma P, et al. Deletion of the p66Shc longevity gene reduces systemic and tissue oxidative stress, vascular cell apoptosis, and early atherogenesis in mice fed a high-fat diet. Proc Natl Acad Sci U S A. 2003; 100:2112-2116.

55. Menini S, lacobini C, Ricci C, Oddi G, Pesce C, Pugliese F, et al. Ablation of the gene encoding p66Shc protects mice against AGE-induced glomerulopathy by preventing oxidant-dependent tissue injury and further AGE accumulation. Diabetologia. 2007; 50:1997-2007.

56. Menini S, Amadio L, Oddi G, Ricci C, Pesce C, Pugliese F, et al. Deletion of p66Shc longevity gene protects against experimental diabetic glomerulopathy by preventing diabetes-induced oxidative stress. Diabetes. 2006; 55:1642-1650.

57. Koch OR, Fusco S, Ranieri SC, Maulucci G, Palozza P, Larocca LM, et al. Role of the life span determinant P66(shcA) in ethanol-induced liver damage. Lab Invest. 2008; 88:750-760.

58. Iurlaro R, Munoz-Pinedo C. Cell death induced by endoplasmic reticulum stress. FEBS J. 2016; 283:2640-2652.

\section{Figures}



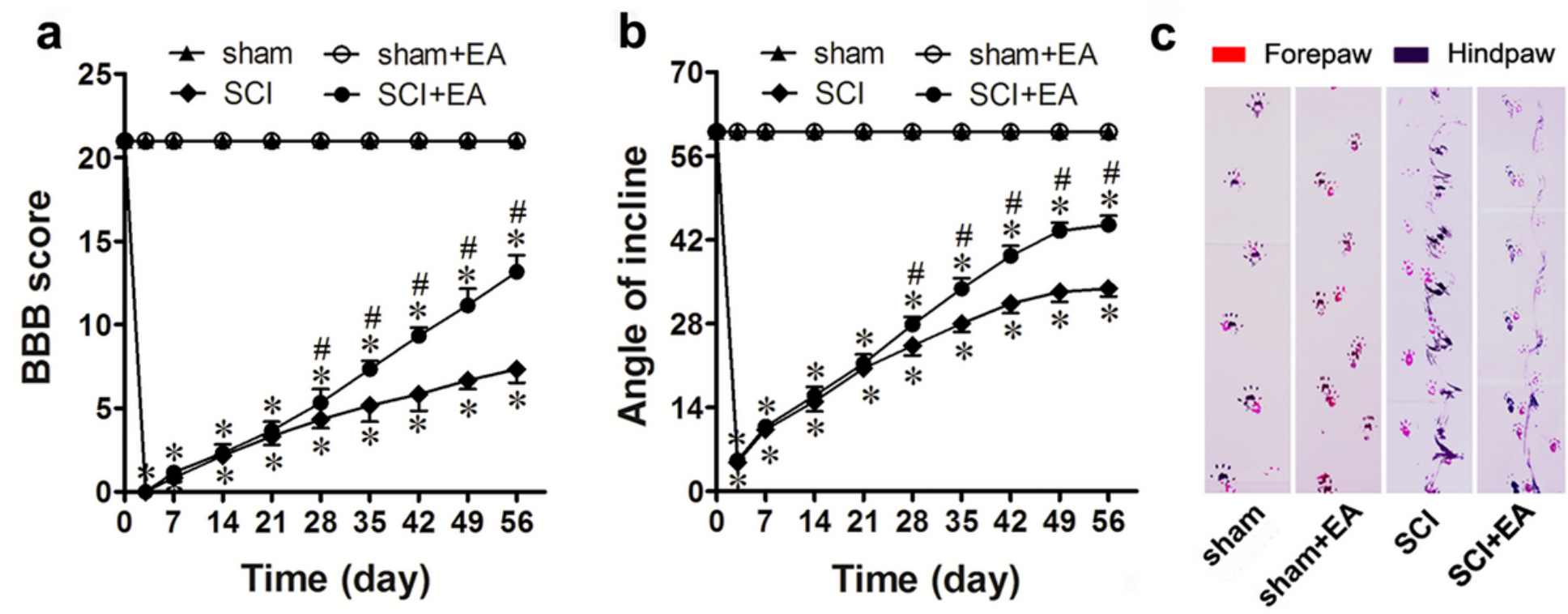

Figure 1

EA treatment improves motor functional recovery after $\mathrm{SCl}$ in rats. $\mathrm{a}-\mathrm{b}$ The $\mathrm{BBB}$ and inclined plane test scores of the sham, sham+EA, SCl, and SCl+EA groups at different time points, respectively. $c$ Rat footprint analysis of the sham, sham+EA, $S C l$, and $S C l+E A$ groups at 56 days after injury. ${ }^{*}<<0.05$ vs. sham group; $\# \mathrm{P}<0.05$ vs. SCl group. 

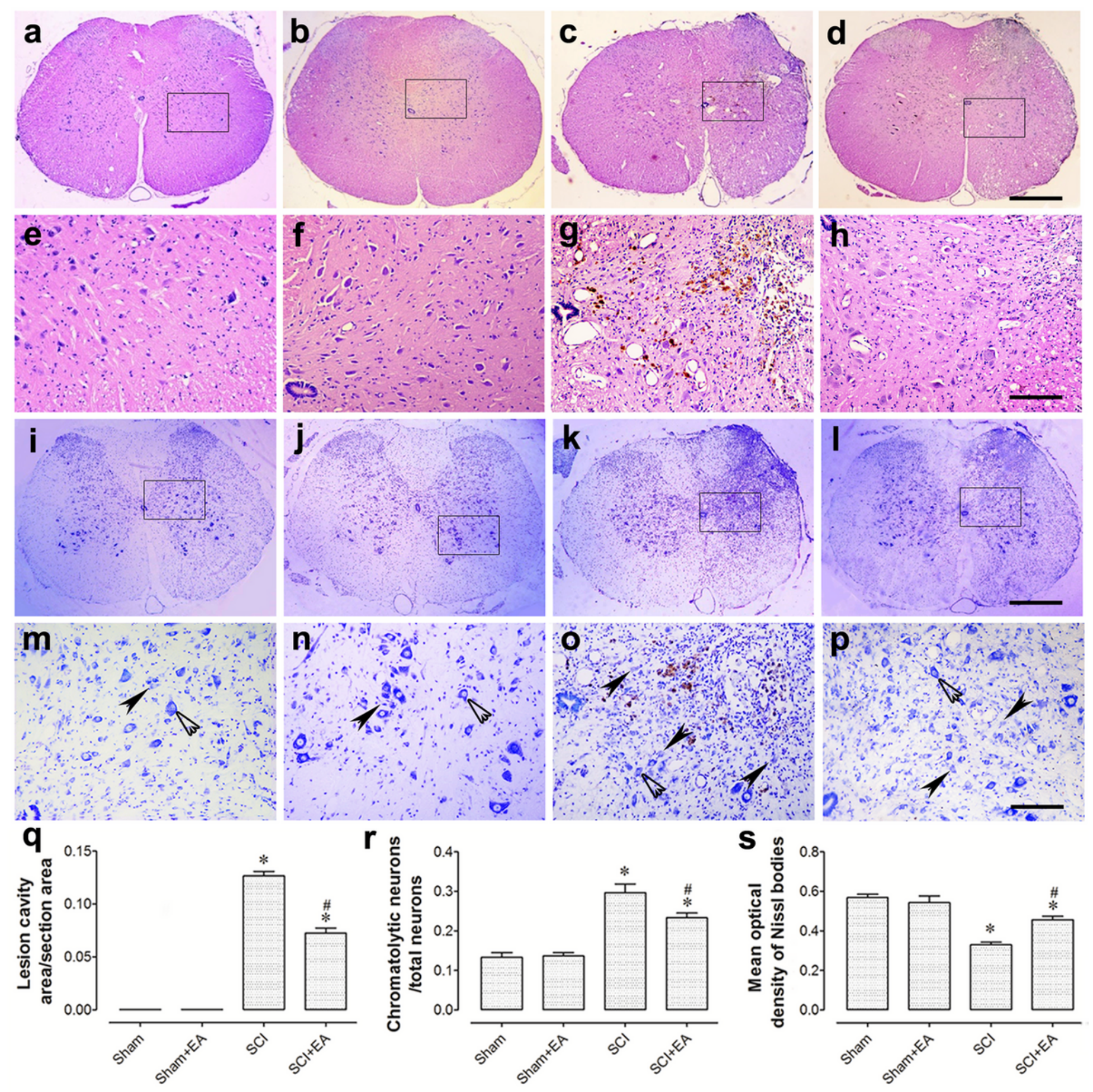

\section{Figure 2}

EA treatment ameliorates histological alterations after $\mathrm{SCl}$ in rats. a-h $\mathrm{HE}$ stained transverse sections of spinal cords from the sham, sham+EA, SCl and SCl+EA groups at 56 days after $\mathrm{SCl}$. i-p The images of Nissl staining of spinal cord sections from the sham, sham+EA, SCl and SCl+EA groups at 56 days after $\mathrm{SCl}$. The normal neurons and chromatolytic neurons are labeled with blank arrowheads and black arrowheads, respectively. Scale bars: $2 \mathrm{~mm}$ in the images with black squares; $100 \mu \mathrm{m}$ in the higher magnification images of the black squares. q Quantification analysis of lesion cavity area in the sham, 
sham+EA, SCl and SCl+EA groups; $r$ Proportions of chromatolytic neurons to total neurons in the sham, sham+EA, SCl and SCl+EA groups. s Comparison of mean optical density (OD) of Nissl bodies of the sham, sham+EA, $\mathrm{SCl}$ and $\mathrm{SCl}+\mathrm{EA}$ groups. ${ }^{*} \mathrm{P}<0.05$ vs. sham group; $\# \mathrm{P}<0.05 \mathrm{vs}$. $\mathrm{SCl}$ group.
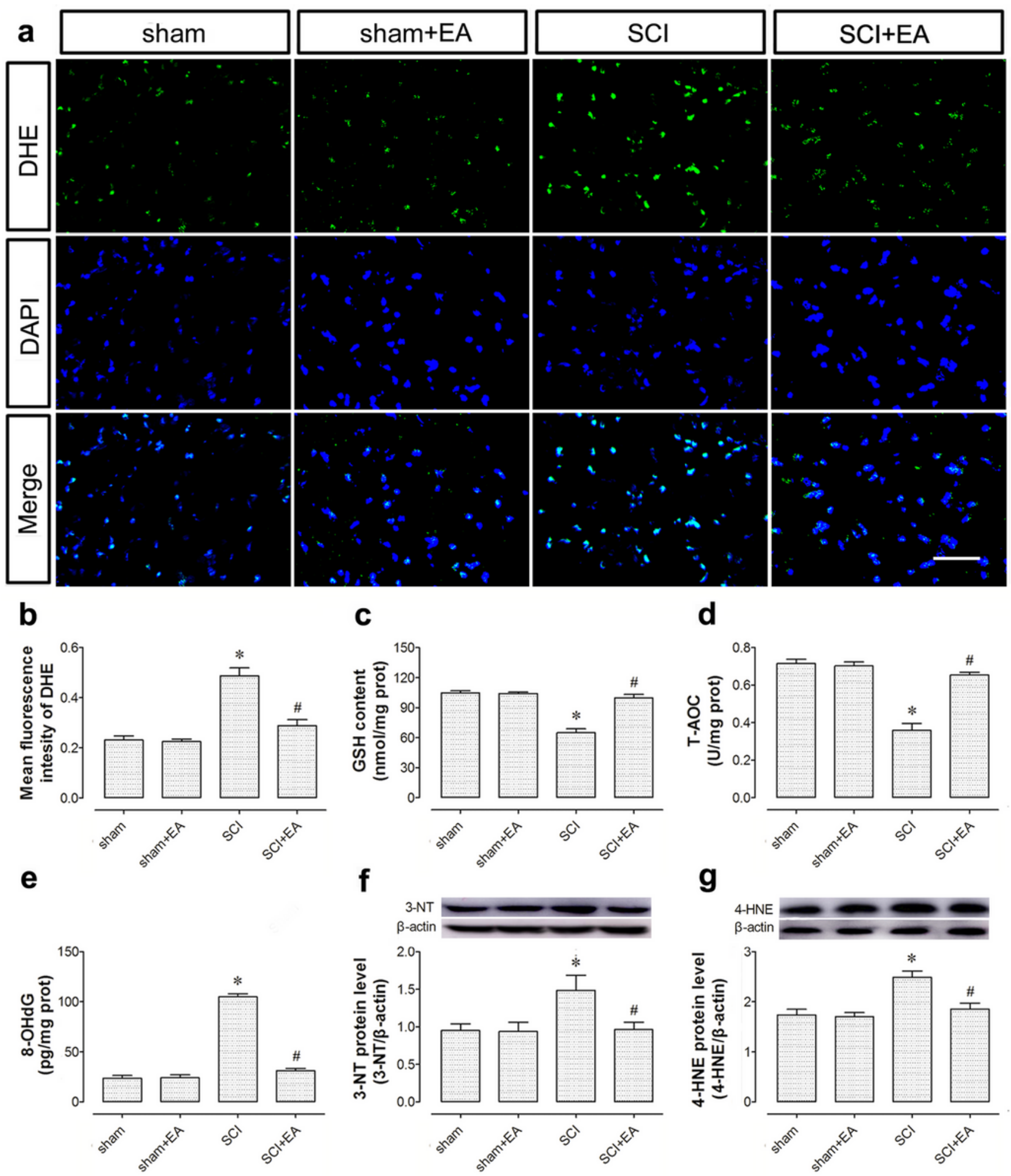

Figure 3

EA treatment alleviates oxidative stress after $\mathrm{SCl}$ in rats. a Representative fluorescent images of ROS stained with DHE in spinal cord tissues from the sham, sham+EA, SCl and SCI+EA groups. Scale bar: 
$100 \mu \mathrm{m}$ in Fig.3a. b Comparison of mean fluorescence intensity of DHE in the sham, sham+EA, SCl and $\mathrm{SCl}+\mathrm{EA}$ groups. C-d Comparison of the levels of GSH and T-AOC in spinal cord tissues of the sham, sham+EA, SCl and SCl+EA groups, respectively. e-g Comparison of the expression levels of 8-OHdG, 3-NT and $4-\mathrm{HNE}$ in spinal cord tissues of the sham, sham+EA, SCl and $\mathrm{SCl}+\mathrm{EA}$ groups, respectively. ${ }^{*}<0.05$ vs. sham group; $\# \mathrm{P}<0.05$ vs. SCl group.
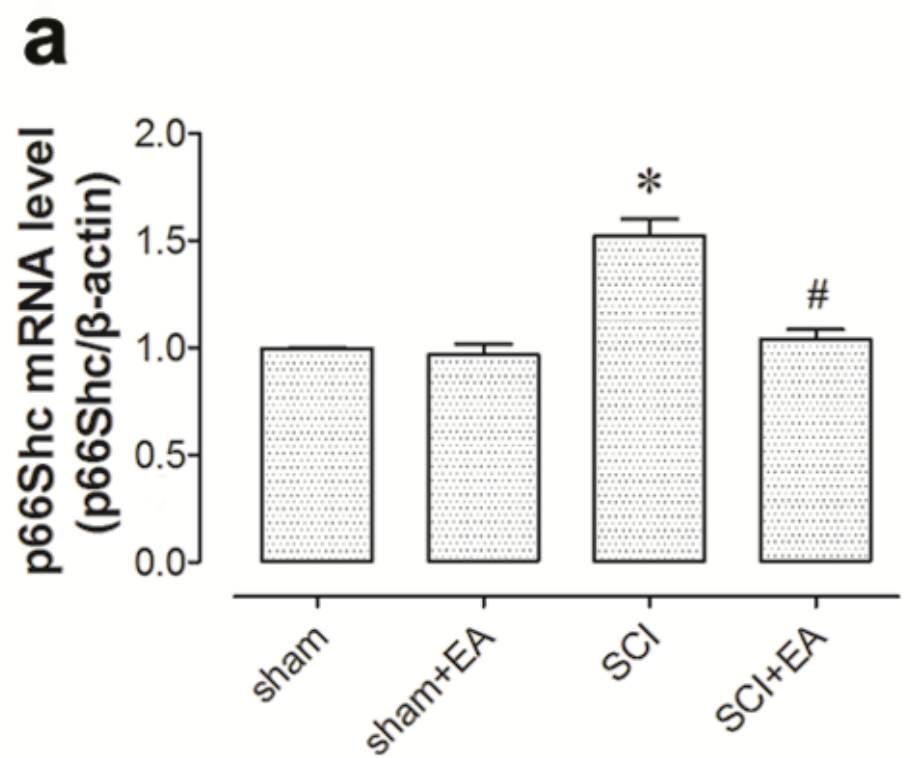

b

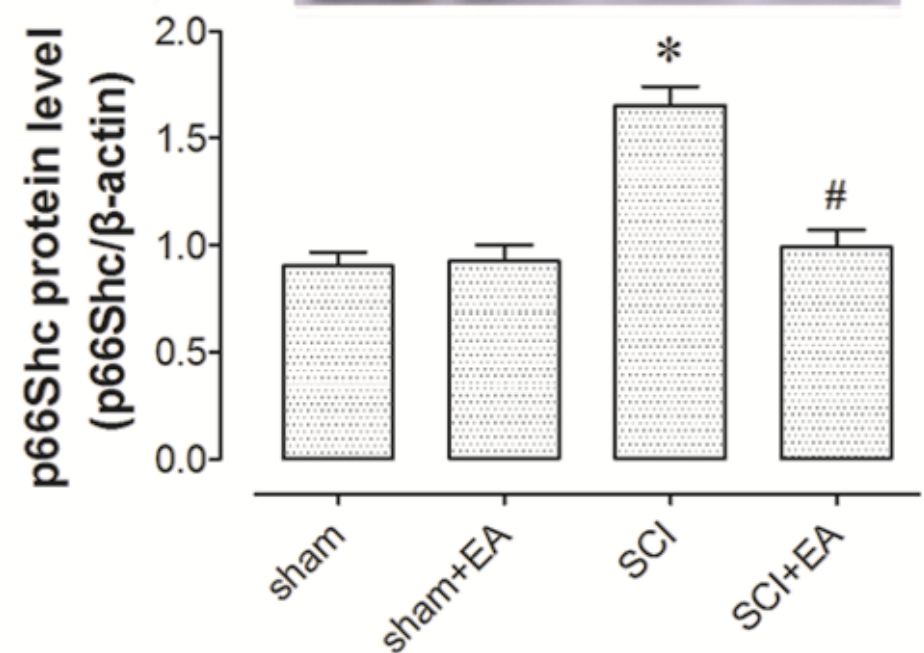

\section{Figure 4}

EA treatment down-regulates p66Shc expression after $\mathrm{SCl}$ in rats. a Comparison of the mRNA expressions of p66Shc in the sham, sham+EA, SCl and SCl+EA groups. b Representative immunoblots (upper) and densitometric analysis (lower) of p66Shc protein expressions in the sham, sham+EA, $\mathrm{SCl}$ and $\mathrm{SCl}+\mathrm{EA}$ groups. ${ }^{*} \mathrm{P}<0.05$ vs. sham group; $\# \mathrm{P}<0.05$ vs. $\mathrm{SCl}$ group. 


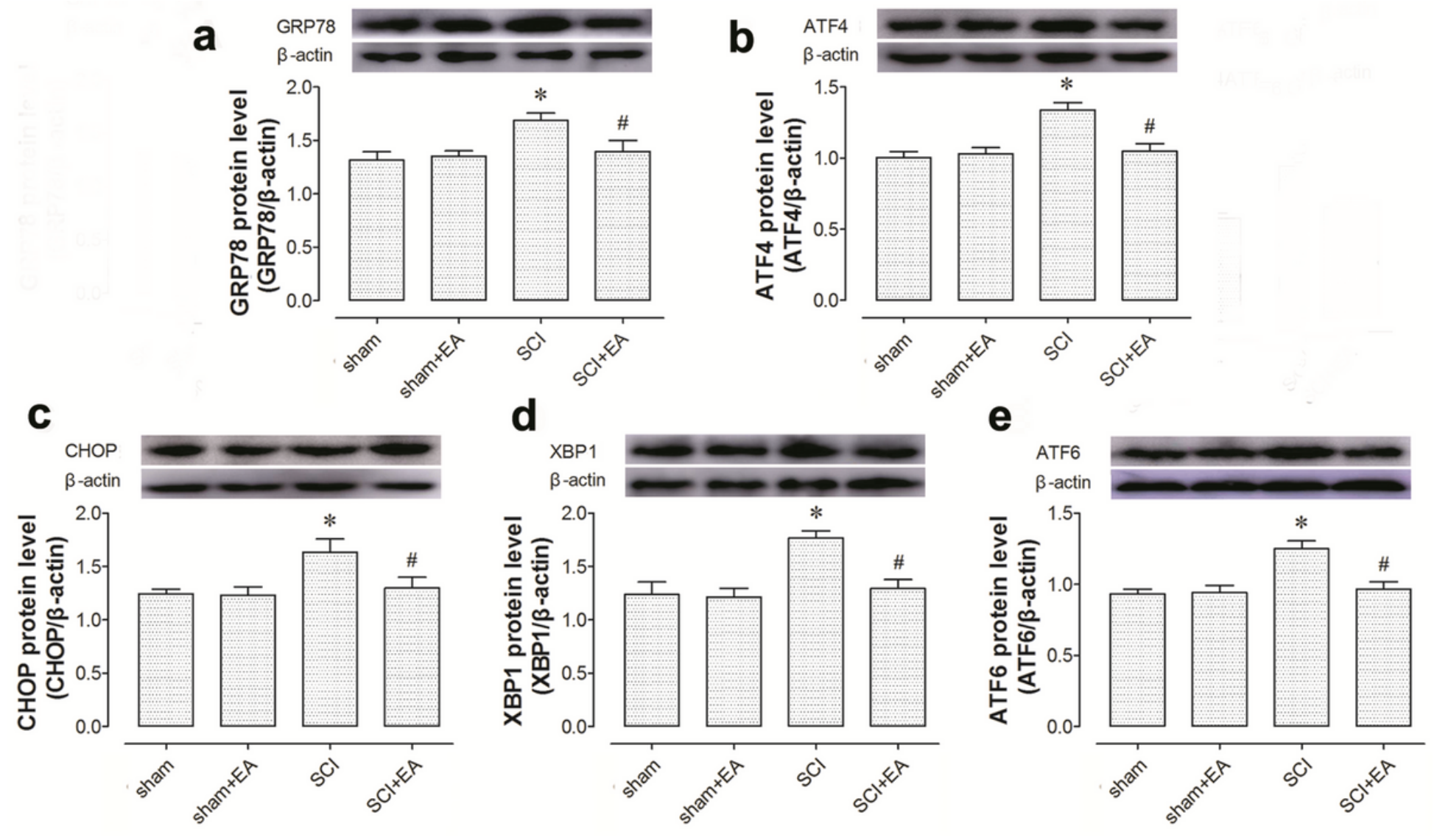

Figure 5

EA treatment suppresses ER stress (ERS) after SCl in rats. a-e Representative immunoblots (upper) and densitometric analysis (lower) of ERS-related proteins GRP78, ATF4, CHOP, XBP1 and ATF6 expressions in the sham, sham+EA, $\mathrm{SCl}$ and $\mathrm{SCl}+\mathrm{EA}$ groups. ${ }^{*} \mathrm{P}<0.05$ vs. sham group; $\# \mathrm{P}<0.05 \mathrm{vs}$. $\mathrm{SCl}$ group. 